\title{
¿Es el artículo una unidad pragmática? Acerca de la teoría de la definitud y la referencia
}

\author{
Is the article a pragmatic unit? \\ On the theory of definiteness and reference
}

\author{
Janusz Pawlik \\ Uniwersytet im. Adama Mickiewicza w Poznaniu (Polonia) \\ pawlik@amu.edu.pl
}

\begin{abstract}
In this paper I intend to review some theories of the article, paying attention to the relations they establish between reference and +/-definiteness of a noun phrase (NP). The prototypes of $+/$-definiteness in Spanish are the definite and indefinite articles and NPs determined by them. Nevertheless, the actual reference of a NP depends mostly on its use (a speech act) and varies according to its pragmatic context. Reference and definiteness are mutually related concepts accompanied by pragmatic and semantic implications. I wish to make a critical overview of some proposals in this field of research.
\end{abstract}

Keywords: article, definiteness, reference, pragmatics, semantics

\section{INTRODUCCIÓN}

En el presente estudio, pretendemos llamar la atención del lector hacia los problemas relativos a la referencia y definitud de los grupos nominales (GN) en relación con el artículo como prototipo de la definitud en español y en otras lenguas. Nos interesarán asimismo los valores semánticos y pragmáticos que ciertos lingüistas atribuyen a la referencia de las expresiones nominales. En nuestra opinión, algunos estudios no interpretan los dos términos de forma satisfactoria (Löbner, 1985). Por ello, en los apartados 6 y 7 presentaremos nuestra postura al respecto. Empezamos, no obstante, con varios 
apartados (2-5) que presentan el estado de la cuestión de los problemas señalados. Nuestra atención se enfoca básicamente en el artículo singular y en el valor no genérico de los GN. En esta contribución dejamos de lado los GN con sentido genérico (Una/La ballena es un mamifero), que se apartan del valor estándar de la definitud y referencia.

\section{EL DILEMA DE LA REFERENCIALIDAD DE LAS EXPRESIONES NOMINALES}

El término referencia tiene un origen filosófico-lógico y fue incorporado a las reflexiones puramente lingüísticas en el marco de la filosofía del lenguaje. Tiene sus raíces en la Antigüedad cuando los filósofos griegos intentaban establecer posibles relaciones entre las palabras y las cosas. El concepto también está conectado con el cálculo lógico de las proposiciones y la evaluación de su veracidad. La problemática de la referencia de las expresiones nominales fue desarrollada por filósofos y lógicos modernos (Frege, Russell, Strawson, Quine).

La función más importante en el esquema de comunicación es la referencial. Referencia, en sentido estrecho, es la capacidad de las expresiones nominales para remitir a entidades de la realidad. En esta acepción la poseen solo: (1) pronombres demostrativos con su valor deíctico, (2) pronombres personales que indican mediante el texto emitido; (3) nombres propios que remiten directamente a objetos y realidades. Estas unidades deben distinguirse de las descripciones definidas ${ }^{1}$ que secundariamente, en posición de argumento, también pueden cumplir una función referencial, p. ej. (Yuri Gagarin) el primer cosmonauta de la historia (Polański, 1993).

En las concepciones referenciales del artículo, el término referencia abarca también las descripciones indefinidas. Su principal exponente es el artículo como uno de los determinantes (referential operators) de un GN. Un sustantivo escueto representa un concepto virtual, esto es, expresa una idea pura y no tiene referencia a la realidad extralingüística. Solo usado con el artículo definido (AD) o indefinido (u otro determinante) viene a adquirir un valor referencial. Un nombre común ligado a un cuantificador general alude a la totalidad de las entidades de su clase (todo hombre es mentiroso), pero junto a un cuantificador específico no remite sino a parte de ella (algún hombre es mentiroso) (Karolak, 1990). Un buen ejemplo de la concepción referencial del artículo es la teoría de la actualización, que se remonta a la gramática de Port-Royal. En el siglo XX fue adaptada por el estructuralismo europeo, en especial por Bally. Muchos estudios españoles sobre el artículo y la definitud de la segunda mitad del siglo pasado se hacen eco de este planteamiento.

Hay autores que se preguntan si la frase indefinida un hombre tiene un carácter referencial. En efecto, no siempre alude a una persona concreta, sino a algún repre-

\footnotetext{
${ }^{1}$ En semántica, los GN con artículo definido.
} 
sentante de la clase denotada por el nombre común (Geach, 1962; Carlson, 2006). En este marco, iniciado por Strawson (1950), la referencialidad caracteriza tan solo a las descripciones definidas (DD), como Compré el coche, con relación a un elemento individual identificado. La identificación unívoca del referente se infiere entonces de la correcta interpretación de la oración y su contexto (extra)lingüístico.

Algunos lingüistas critican la conclusión general de que las descripciones indefinidas carezcan de referencialidad. Al lado de claros usos no referenciales en posición de atributo (Soy un hombre) ${ }^{2}$, Kleiber (1981) enumera usos en contextos reconocidos como no transparentes (opacos), en los que los GN indefinidos muestran una lectura ambigua (spécifique non spécifique), es decir, en algunos casos pueden indicar a un individuo con referencia unívoca (se tiene a alguien real en la mente), pero en otros no, p. ej.:

Paul veut épouser une Tahitienne.

Pierre essaye de trouver un psychanaliste [cualquiera].

Solo la primera interpretación es considerada como referencial. Es una postura pragmática en la que el punto de mira lo constituye el hablante, quien se está refiriendo a alguien en concreto. Actualmente, un planteamiento pragmático parecido respecto al concepto de referencia lo representa Lyons (1999), quien declara:

I shall continue here to speak informally of both simple definites and indefinites as potentially referring, but without commitement as to whether the reference is semantic or pragmatic. In characterizing indefinites like a car (in I bought a car) as referring, I mean no more than that there is a particular object which the speaker is thinking of as motivating the choice of description (p. 166).

En este marco, se sitúan también otros autores anglosajones (Donnellan, 1978; Wilson, 1978; Fodor, Sag, 1982), quienes distinguen entre el uso específico del nombre común a car (en I bought a car) como expresión referencial, y el uso inespecífico del nombre a book (en Pass me a book) como expresión no referencial. De modo que igualan la lectura inespecífica (non-specific) con la no referencial (non-referential) y específica (specific) con la referencial (Lyons, 1999). Laca (1999), Leonetti (1990) y ASALE (2009) identifican la referencia con el carácter específico de las expresiones nominales y aluden a dos principales rasgos de la especificidad: la existencia del objeto y su identificabilidad por parte del hablante.

Conviene destacar que hay filósofos y lógicos que defienden una postura extrema (Kripke, 1977; Neale, 1990; Karolak, 1990). Consideran que todas las descripciones indefinidas son de por sí no referenciales, afirmando que la capacidad referencial les pertenece tan solo a los nombres propios, demostrativos y pronombres personales. Se

${ }^{2}$ En esta posición las descripciones definidas tampoco refieren, p. ej. Napoleon was the greatest French soldier (Strawson, 1950). 
excluyen, en principio, también las DD (descripciones definidas). La referencia sensu stricto tiene en este enfoque un carácter asemántico (referencia directa) (Kripke 1977)3. Una de las ventajas de este planteamiento consiste en evitar la polémica en torno a la referencialidad de los nombres vacíos o inespecíficos, p. ej. Naturalmente, el futuro Presidente tomará precauciones para impedirlo / ¿Encontraré una esposa semejante a Leda?

\section{EN BUSCA DE LA ESENCIA DE LA DEFINITUD}

El problema de la referencia de las expresiones nominales es distinto del de la definitud. Sin embargo, como advierte Leonetti (1990), ambos conceptos quedan prácticamante asimilados en muchas lenguas y tienden a confundirse en la tradición gramatical. Filósofos y lógicos se ocuparon al principio de la referencia y no de la definitud. En las lenguas articuladas, el prototipo de definitud es el artículo junto con el GN al que determina. La búsqueda de la esencia de la definitud empieza con consideraciones de naturaleza filosófico-lógica. Las observaciones que nos proponemos hacer en materia de referencia y definitud tienen plena validez en la lengua española.

Generalmente se admiten como definidas las expresiones nominales precedidas del AD. ¿Pero es adecuado este planteamiento? Karolak (1989) pone en tela de juicio la tesis según la cual el $\mathrm{AD}$ en su acepción no genérica funciona siempre como marca de definitud del objeto denotado. Se sirve al respecto del esquema preposicional [el $\mathrm{N}$ de un $\mathrm{N}$, p. ej. la capitale d'un pays européen, y concluye que "[d]es descriptions telles que la capitale d'un pays européen sont de façon evidente indefinies (non uniques), néanmoins l'article defini y est obligatoire. Il s'ensuit que celui-ci est fonction de la completude sémantique de la proposition". Queda por comentar aún el estatuto de los GN en sentido genérico, que se apartan del valor estándar de la definitud, y que, por ello, dejamos de lado en esta contribución.

\section{4. +/-DEFINITUD EN TANTO OPOSICIÓN BINARIA ${ }^{4}$}

A continuación pasamos a comentar algunos rasgos de carácter binario (presencia/ ausencia) que fueron propuestos por investigadores como condición de la definitud nominal. Empezamos con el principio de unicidad que aparece por primera vez en la teoría de las descripciones del filósofo británico Russell (1905). Según ella, se permite la ocurrencia de sintagmas nominales definidos en el contexto de una oración cuando uno y solo un objeto encaja en su contenido descriptivo, p. ej. El rey de Francia es

\footnotetext{
${ }^{3}$ Un nombre propio o un deíctico (este (vaso), aquel (ordenador), etc.) es identificable con su referente sin ayuda de su contenido descriptivo (directly referring).

${ }^{4}$ Recuérdese que existen propuestas de clasificación de las expresiones nominales no en términos de oposición binaria (+/-definitud), sino como portadoras de diferentes grados de contenido informativo.
} 
calvo. La condición de unicidad propugnada por Russell fue aceptada con amplitud por gramáticos modernos -incluidos los españoles- como el principal criterio para delimitar la definitud. Con este concepto está vinculado el carácter identificable del objeto denotado para el receptor (Strawson, 1950; Searle, 1969).

Otra teoría entiende la definitud como familiaridad del oyente con el referente aludido (Christopherson, 1939), apoyada en el contraste 'consabido' 'no consabido' . Una propuesta análoga en el área romance es la théorie de la notoriété de Damourette \& Pichon (1927-1950). Sin embargo, las teorías apoyadas en la tesis de la familiaridad fallan en varias ocasiones, algo que advierte el propio Christopherson (1939, pp. 99100): "This term [= familiarity] is not always quite correct. (...) Talking of a certain book, it is perfectly correct to say The author is unknown". Heim (1982) desarrolla de forma original y creativa las ideas sobre la familiaridad, y su estudio se centra en el uso anafórico de los GN definidos.

Löbner (1985) adopta una postura opuesta a Heim, en la que el uso prototípico de los GN definidos no es el anafórico sino el funcional ${ }^{5}$ (principio de funcionalidad). El $\mathrm{AD}$ anuncia que la expresión remite sin ambigüedad a un solo objeto debido a su contenido funcional (uno a uno). Un concepto funcional es, por ejemplo, ser el alcalde de una pequeña ciudad (como se sabe, alcalde no hay más que uno). Cuando el GN no indica un concepto funcional, se necesitan otros recursos para establecer el referente de forma no ambigua (Leonetti, 1996).

Otros intentos de descubrir una variable única y segura capaz de imponer + /-definitud a un GN giran en torno a las teorías pragmáticas basadas en el concepto de 'objeto saliente'. Esta perspectiva es defendida por Lewis, que rechaza abiertamente la condición de unicidad ideada por Russell. Lewis (1979) busca el meollo de la definitud en el principio de prominencia: "The proper treatment of description must be more like this: 'the $\mathrm{F}$ denotes $\mathrm{x}$ if and only if $\mathrm{x}$ is the most salient $\mathrm{F}$ in the domain of discourse, according to some contextually determined salience ranking" (p. 178).

\section{REFERENCIA Y DEFINITUD: ¿CONTRADICCIÓN O AFINIDAD?}

En la teoría de Strawson (1950), la proposición es verdadera siempre que tiene referencia. La lógica y la filosofía evalúan solo la veracidad de las oraciones enunciativas, mientras que una lengua natural dispone también de muchas otras oraciones; de modo que el concepto de referencia de un GN debe tomar en cuenta criterios y condicionamientos propiamente lingüísticos.

Muchos lingüistas asimilan el concepto de referencia con el de definitud. No obstante, como se sabe, algunos gramáticos y semánticos consideran referenciales también las descripciones indefinidas. Pero tampoco todas las DD poseen un carácter

${ }^{5} \mathrm{El}$ concepto se entiende de forma distinta a la que propusieron Martinet o Coseriu. 
referencial en su uso (Donnellan, 1966). Las descripicones definidas suelen emplearse incluso en el caso de faltar referentes en el mundo real. Lo observamos, p. ej., en oraciones hipotéticas del tipo ing. the murderer of Smith would have to be insane, fran. les enfants éventuels des Dupont hériteraient une fortune, esp. el concursante ganador obtendrá un viaje al Caribe (Polański, 1993). Es un problema que acapara desde antiguo la atención de filósofos del lenguaje. Bertolet (1984) sostiene que personajes ficticios (Pegasus, Sancho Panza) existen en mitos, leyendas o cuentos y son comúnmente identificables. Más aún, la ampliación del concepto de referencia a todas las DD nos acerca a la auténtica función representativa del lenguaje. Recogiendo la opinión de Kamp \& Reyle (1993), Carlson (2006) advierte que "the reference of language is not objects in the real world, but rather DISCOURSE ENTITIES" (p. 96). En efecto, en función de cómo se entiende la referencia de las expresiones nominales, el término se extiende a un mayor o menor número de sus representantes.

A continuación, vamos a esbozar de forma esquemática la relación entre referencia y definitud establecida en algunas teorías en torno al artículo. Se incluirán conjuntos que representan los usos de descripciones definidas (DD), indefinidas (DI), nombres propios (NP), pronombres personales (PP) y pronombres demostrativos (PD), junto con su correspondiente alcance referencial (con el signo "denotamos que solo parte de un conjunto de expresiones es capaz de referir). Así, daremos cuenta de las cuatro teorías del artículo comentadas previamente:

(1) Teorías referenciales [DD + DI + NP + PP + PD].

(2) Teorías referenciales reducidas [DD' + DI' $+\mathrm{NP}+\mathrm{PP}+\mathrm{PD}]$.

(3) Teoría inspirada en las ideas de Strawson [DD + NP + PP + PD].

(4) Teoría lógicista $[\mathrm{DD}\rangle+\mathrm{NP}+\mathrm{PP}+\mathrm{PD}]$.

Es de notar que el primero de los modelos comprende en el grupo de DD y DI también sus usos genéricos. Los tres restantes pasan por alto este valor al considerarlo no referencial (cuantificacional). Tampoco se asigna referencialidad, en todas las doctrinas, a los usos nominales atributivos. Por nuestra parte, abogamos por el tercer planteamiento como el más coherente y claro, aunque poco difundido. Rechazamos al mismo tiempo las concepciones extremas 'negacionistas', que mantienen que ninguna expresión nominal sea capaz de presentar una referencia objetiva (Quine, 1972).

\section{ASPECTOS PRAGMÁTICOS Y SEMÁNTICOS DE LAS DESCRIPCIONES DEFINIDAS}

Ya Strawson (1950) relacionó la referencia con el uso de una proposición en un acto de habla, es decir, le concede una dimensión pragmática. Así, el GN el rey de Francia muestra un valor referencial solo con respecto a un pasado lejano, de modo 
que la proposición el rey de Francia es calvo pronunciada hoy no se puede evaluar en cuanto a su veracidad. De igual modo el hombre con el pelo canoso se asigna a una persona particular únicamente en una situación dada. Desde un punto de vista semántico, esta expresión no tiene una capacidad automática para indicar un único objeto identificable fuera del contexto. Definida así, la referencialidad es también rasgo de algunas unidades nominales (pronombres personales, demostrativos y algunos nombres propios como Juan, González).

Las descripciones definidas (DD) se dividen en 'completas' e 'incompletas'. Las primeras son unívocas y pueden existir fuera del entorno lingüístico-situacional, p. ej. el Primer Ministro de Gran Bretaña, el tren más rápido, el año 1984. Las descripciones incompletas, menos informativas, adquieren el rasgo de unicidad (no ambigüedad) solo en conexión con un contexto y en una situación determinada: el representante, la persona que llamó anoche, el coche rojo, etc. Según Abbott (2019), Löbner (1985) llama semantic definites a las descripciones completas, mientras que a las incompletas las denomina pragmatic definites.

Sin embargo, el lingüista alemán lleva a cabo una división más compleja: "In (...) 'semantic definites' the referent of the definite is established independently of the immediate situation or context of utterence. (...) 'Pragmatically definite' NPs, on the other hand, are essentially dependent on special situation and contexts for the non-ambiguity of a referent" (1985, p. 298). En su extenso artículo, Löbner cita las frases the fattest wrestlers, the post office, the first man como ejemplos de definidos semánticos. Por supuesto, el mismo hecho de la definitud formal de estas expresiones no se traduce en su carácter referencial, tal como sucede en los nombres propios. La definitud semántica es interpretada por Löbner como 'unicidad funcional' y no tiene nada que ver con la referencialidad inherente de un GN definido. Y eso no le preocupa, ya que el autor desatiende el factor de referencia. Solo mediante el aumento de informatividad de dichos GN se pueden convertir en expresiones unívocamente referenciales (uniquely referring, según Strawson), es decir, the post office in Sherwood, the first man in space. Quedan por discutir todavía los GN que poseen de por sí una unicidad funcional, pero son de hecho expresiones indefinidas (the Mayor of a town in Wales $^{6}$ ). En consecuencia, no se pueden contar ni entre los definidos semánticos ni los pragmáticos. En expresiones definidas por excelencia con el esquema binominal $N$ of $N(N$ de $N)$, el primer nombre se une a menudo a su modificador siguiendo el principio funcional (uno a uno): p. ej. the headmaster of the school (cada escuela tiene un director). Y es lo que le otorga al conjunto un carácter semántico. Es de notar que, curiosamente, también hasta los nombres propios y pronombres personales, semánticamente vacíos, se catalogan en Löbner como semánticos (pp. 299-300). En este modelo son definidos pragmáticos todos los que no cumplen el requisito de unicidad funcional expuesta supra: the tall boy, the man with gray hair, etc. Es de señalar que

${ }^{6}$ Una ciudad en Gales tiene solo un alcalde, pero hay muchos alcaldes en Gales. 
todas las construcciones con oraciones de relativo (the man I met yesterday, the woman Bill came out with last night, etc.) quedan calificadas siempre como definidos pragmáticos.

Inspirados por la distinción entre descripciones completas e incompletas y otras parecidas (Löbner, 1985; Rothschild, 2007), nos proponemos esbozar un esquema de clasificación de las DD. Nuestra propuesta consistirá en realizar un reparto más sencillo, pero añadiendo el parámetro adicional 'referencia': los definidos semánticos se sustituyen por descripciones con referencia semántica (DRS) y los definidos pragmáticos por descripciones con referencia pragmática (DRP). De modo que presentamos el aspecto pragmático y semántico de la definitud basada en la noción de referencia. Las DRS estarán provistas siempre de AD sin atender al contexto de uso, mientras que las DRP englobarán tanto los GN precedidos siempre del AD como aquellos que en entornos oportunos exigirían un AI. Siendo así, el grupo de definidos pragmáticos se puede subdividir en dos subcategorías adicionales: definidos pragmáticos obligados y definidos pragmáticos potenciales. Como resultado, dos de los conjuntos nuevos abarcarán expresiones dotadas siempre de definitud formal y el tercero, aquellas con posible alternancia del artículo según el contexto.

Tabla 1. Descripciones definidas (Elaboración propia)

\begin{tabular}{|l|l|l|}
\hline \multirow{2}{*}{$\begin{array}{l}\text { Descripciones con referencia } \\
\text { semántica }\end{array}$} & \multicolumn{2}{|l|}{ Descripciones con referencia pragmática } \\
\cline { 2 - 3 } & Definidos pragmáticos obligados & $\begin{array}{l}\text { Definidos pragmáticos poten- } \\
\text { ciales }\end{array}$ \\
\hline $\begin{array}{l}\text { (El hombre que mató a M. L. } \\
\text { King; la Tierra; el primer cosmo- } \\
\text { nauta de la historia })\end{array}$ & $\begin{array}{l}\text { (los luchadores más gordos; el el que naci; el padre de este } \\
\text { joven })\end{array}$ & $\begin{array}{l}\text { (el perro; el hombre con gue me encontré ayer en } \\
\text { el cine })\end{array}$ \\
\hline
\end{tabular}

En nuestra propuesta, los sustantivos aislados pertenecen en su aplastante mayoría al tercer grupo con la posible alternancia del artículo, p. ej. perro, hombre, oro, investigación. En estos casos se adquiere definitud debido a la anáfora directa (Entra un hombre. El hombre pide una cerveza), asociativa (Preparo un artículo. Pronto te envio el borrador), así como al contexto situacional ( $L$ a ciudad [en que estamos] es grande y bella). Los GN complejos, en función de su informatividad, serán siempre definidos en los referenciales semánticos debido a su unicidad absoluta: El hombre que mató a M. L. King; la palabra 'bueno'; El Japó; y en los definidos pragmáticos obligados, que son referenciales en un contexto: los luchadores más gordos; el día en que nací; el padre de este joven. Una parte de las descripciones con referencia pragmática (definidos pragmáticos potenciales) serán variables en su artículo (definitud potencial) y, además de GN simples, recogerán también grupos complejos: el/un hombre que vino ayer, el/un hombre con gafas, el/un hijo de Pedro. El AD como marca de unicidad referencial aparece solo en determinados contextos. Considérese el ejemplo 
el hombre que vino ayer, nombrado por primera vez, que será la forma esperada en una situación con poca afluencia de gente, p. ej. en un hogar familiar; no obstante, esta misma expresión tomará la marca de indefinitud en una charla entre empleados de una oficina de correos. La identificación pragmática, marcada con AD, se lleva a cabo si se establece la conformidad unilateral entre el referente y la situación. En el caso de referentes plurales es mejor hablar de inclusividad que de su unicidad, siguiendo la propuesta de Hawkins (1978).

Nos resta señalar un cuarto conjunto de descripciones definidas, las que no presentan referencia (descripciones no referenciales), según algunas teorías reduccionistas. Como se sabe, su definitud consiste en la identificación por parte del oyente de una entidad designada vacía; en este conjunto se incluye una amplia variedad de GN, como el futuro Presidente de EEUU; el posible asesino de Smith, la ciudad de Gotham, etc.

\section{OBSERVACIONES FINALES}

En el esquema presentado arriba hemos pretendido relacionar en un sistema homogéneo los conceptos examinados en este artículo: definitud, referencia y los polémicos términos semántico y pragmático relativos a las DD. No es el primer intento ni, seguramente, el último de reflexión detallada sobre las DD completas/incompletas. Pero es diferente en contenido y algunos términos de los planteamientos de Rothschild (2007), Peacocke (1975) o Donnelan (1966), quienes también han investigado esta problemática.

La división bipartita expuesta en los autores aludidos no refleja de lleno el funcionamiento del artículo en GN concretos, es decir, algunas DD semánticas/completas pueden llevar AI (un presidente, un primer ministro, una oficina de correos, etc.), así como las DD pragmáticas/incompletas pueden alternar este determinante (un coche rojo, una chica que me interesa). Nuestro esquema se propone ordenar las DD en dos conjuntos que toman obligatoriamente $\mathrm{AD}$ y el tercero que puede alternar su forma con AI. Esta información es de suma importancia para estudiantes de ELE, especialmente aquellos cuya lengua materna carece de artículo.

Como ya se ha dicho, los definidos semánticas de Löbner, como el primer ministro, aseguran la unicidad lógica (funcional), pero no la referencial. Esta observación no es muy reveladora y los autores citados pasan por alto deliberadamente el parámetro de referencia. En nuestra propuesta aparece este concepto como un factor adicional importante. Así, para asegurarle al GN citado la calidad de definido semántico sensu stricto (con referencia semántica) deberíamos añadirle adjuntos de tiempo y lugar (en 1996, de España, etc.). Somos conscientes de que los GN de este tipo (informativos al máximo) son calificados por algunos autores como 'actualizados', pero el término nos parece inoportuno por ser aplicable asimismo a descripciones indefinidas (un hombre, unos perros). 
El requisito de dotar tales GN de más información lleva a restringir notablemente el número total de $D D$ con referencia semántica comparado con el de los definidos semánticos de Löbner. Por otro lado, parte de los GN con modificadores oracionales, en Löbner y Rothschild catalogados como DD pragmáticas (o particularizadas), pasarán a las nuevas descripciones semánticas, p. ej. El hombre que mató a M. L. King como una expresión unívocamente referencial. Repartimos así entre conjuntos distintos los GN modificados por una oración de relativo generadores de definitud obligada (conjuntos 1,2) y posible (conjunto 3). Esta variación falta en los autores comentados.

Para terminar, conviene señalar que el presente estudio se ciñe básicamente a aquellos contextos que se caracterizan por un alto grado de similitud con la lengua inglesa. Por consiguiente, se pasan por alto aquellas situaciones contextuales, propias de la lengua española, donde los valores pragmáticos del artículo están mejor representados, como en el atributo del predicado nominal (cf. Juan es conductor vs. Juan es un conductor vs. Juan es el conductor). 


\section{BIBLIOGRAFÍA}

Abbott, B. (2006). Definite and indefinite. In K. Brown (Ed.), Encyclopedia of language and linguistics (pp. 392-399). Amsterdam: Elsevier.

Abbott, B. (2019). The indefiniteness of Definiteness. In J. Gundel, \& B. Abbott (Eds.), The Oxford Handbook of Reference (pp. 121-135). Oxford: Oxford University Press. DOI: 10.1093/oxford$\mathrm{hb} / 9780199687305.013 .8$.

ASALE (2009). Nueva Gramática de la lengua española. Madrid: Espasa.

Bertolet, R. (1984). Reference, fiction and fictions. Synthèse, 60, 413-37.

Carlson, G. (2006). Reference. In L. Horn, \& G. Ward (Eds.), The Handbook of Pragmatics (pp. 74-96). Malden, MA: Blackwell Publishing.

Christopherson, P. (1939). The Articles: a Study of their Theory and Use in English. Copenhagen: Munksgaard.

Donnelan, K. (1966). Reference and definite descriptions. Philosophical Review, 77, 281-304.

Geach, P.T. (1962). Reference and Generality. New York: Cornell University Press.

Heim, I. (1982). The Semantics of Definite and Indefinite NP's. Amherst: University of Massachussetts.

Karolak, S. (1989). L'article et la valeur du syntagme nominal. Paris: PUF.

Karolak, S. (1990). Kwantyfikacja a determinacja w językach naturalnych. Warszawa: PWN.

Kleiber, G. (1981). Problèmes de référence: descriptions définies et noms propres. Paris: Klincksieck.

Kripke, S. (1977): Speaker's reference and semantic reference. In P.A. French, T.E. Uehling, Jr. \& H. Wettstein (Eds.), Midwest Studies in Philosophy, vol. II: Studies in the philosophy of language (pp. 255-276). Morris, MN: University of Minnesota.

Laca, B. (1999). Presencia y ausencia de determinante. In I. Bosque, \& V. Demonte (Eds.), GDLE (pp. 891-928). Madrid: Espasa-Calpe.

Leonetti Jungl, M. (1990). El artículo y la referencia. Madrid: Taurus.

Leonetti Jungl, M. (1996). El artículo definido y la construcción del contexto. Signo \& Seña, 5, 102-136.

Lewis, D. (1979). Scorkeeping in a language game. Journal of Philosophical Logic, 8, 339-359.

Löbner, S. (1985). Definites. Journal of Semantics, 4, 279-326.

Lyons, C. (1999). Definiteness. Cambridge: Cambridge University Press.

Neale, S. (1990). Descriptions. Cambridge, MA: MIT Press.

Polański, K. (Ed.) (1993). Encyklopedia językoznawstwa ogólnego. Wrocław: Ossolineum.

Quine, W.V. (1972). Méthode de logique. Paris: Armand Colin.

Rothschild, D. (2007). Presuppositions and scope. Journal of Philosophy, 104, 71-106.

Russell, B. (1905). On denoting. Mind, 14, 479-493.

Searle, J. (1969). Speech Acts. Cambridge: Cambridge University Press.

Strawson, P.F. (1950). On referring. Mind, 59, 320-344. 